\section{IMMUNE PROFILING REVEALS ENRICHMENT OF DISTINCT IMMUNE SIGNATURES IN HIGH-RISK ORAL POTENTIALLY MALIGNANT DISORDERS}

${ }^{1}$ Chai Gan*, 'Bernard Kok Bang Lee, ${ }^{2}$ Shin Hin Lau, ${ }^{3}$ Thomas George Kallarakkal, ${ }^{3}$ Zuraiza Mohamad Zaini, ${ }^{4}$ Rosnah Binti Zain, ${ }^{2}$ Hans Prakash Sathasivam, ${ }^{5}$ Joe Poh Sheng Yeong, ${ }^{6}$ Natalia Savelyeva, ${ }^{6}$ Gareth Thomas, ${ }^{7}$ Christian Ottensmeier, ${ }^{3}$ Hany Ariffin, ${ }^{1}$ Sok Ching Cheong, 'Kue Peng Lim. 'Cancer Research Malaysia, Subang Jaya, Malaysia; ${ }^{2}$ Institute for Medical Research, Kuala Lumpur, Malaysia; ${ }^{3}$ University of Malaya, Subang Jaya, Malaysia; ${ }^{4}$ MAHSA University, Jenjarom, Malaysia; ${ }^{5}$ Institute of Molecular Cell Biology, Singapore, Singapore; ${ }^{6}$ University of Southampton, Southampton, UK; ${ }^{7}$ University of Liverpool, Liverpool, UK

Background Patients with oral potentially malignant disorders (OPMD) having moderate or severe oral epithelial dysplasia (OED) have a greater risk of developing oral squamous cell carcinoma (OSCC) compared to mild OED with an odds ratio of 2.4. ${ }^{1}$ The involvement of specific immune cell types associated with malignant transformation have been reported, giving rise to clinical trials in immunoprevention. However, the immune landscape of OPMD remains understudied. In this study, we aimed to elucidate the immune landscape of highrisk OPMD by transcriptomic profiling for the identification of potential immunoprevention strategy.

Methods Histological evaluation was performed on hematoxylin and eosin (H\&E)-stained tissues to investigate the differences of lymphocyte infiltration in benign lesions $(n=16)$, highrisk OPMD consisted of moderate and severe OED $(n=46)$ and early-stage OSCC $(n=6)$. Formalin-fixed paraffinembedded tissue sections of selected cases from each sample type were subjected to RNA sequencing. Weighted-gene-correlation network analysis (WGCNA) was used to identify key gene modules expressed in specific disease type. ${ }^{2}$ The immune landscape of high-risk OPMD was elucidated by the enrichment of immune signatures using single-sample gene set enrichment analysis. ${ }^{3-5}$ The response of high-risk OPMD to anti-PD1 treatment was predicted by the detection of T-cellinflamed condition. ${ }^{6}$ Validation was performed by multiplex immunofluorescent (mIF) staining.

Results Our H\&E evaluation showed that lymphocyte infiltration into the epithelial was seen in $80 \%$ of high-risk OPMD and early-stage OSCC, compared to $9 \%$ of benign lesion. Gene modules identified from WGCNA analysis revealed that genes involved in immune-related pathways were overexpressed in high-risk OPMD and in early-stage OSCC when compared to benign lesion, but unchanged between high-risk OPMD and early-stage OSCC. We further demonstrated that immune signatures representing lymphocyte infiltration, MHCI antigen presentation and cytotoxic immune responses were enriched in high-risk OPMD, indicating the presence of immune surveillance. High-risk OPMD can be grouped into the T-cell-inflamed and non-immune reactive subtypes. The Tcell-inflamed subtype is enriched with $\mathrm{T}$ cells, interferon signaling and PD-1/PD-L1 immune checkpoint proteins, suggesting that these lesions may be amenable to anti-PD1 treatment. Meanwhile, the non-immune reactive subtype demonstrated low enrichment in signatures for immune cell infiltration, indicating a need of intervention to induce lymphocyte infiltration. Using mIF staining, we observed an increase of CD45+ immune cell population expressing PD-L1 in high-risk OPMD. Conclusions Immune surveillance is a prominent feature of high-risk OPMD. However, different subsets of high-risk OPMD exist, suggesting a need of different immunoprevention approaches to prevent disease progression which warrants further investigation.
Acknowledgements This study was supported and funded by the Global Challenge Research Fund by the Medical Research Council, UK (MR/P024351/1) and Cancer Research Malaysia. We thank the Ong Heng Tiang \& Ong Sek Pek Foundation for scholarship sponsorship.

\section{REFERENCES}

1. Iocca O, Sollecito TP, Alawi F, et al. Potentially malignant disorders of the ora cavity and oral dysplasia: a systematic review and meta-analysis of malignant transformation rate by subtype. Head Neck 2020;42:539-55.

2. Langfelder P, Horvath S. WGCNA: an R package for weighted correlation network analysis. BMC Bioinformatics 2008:9:559.

3. Subramanian A, Tamayo P, Mootha VK, et al. Gene set enrichment analysis: a knowledge-based approach for interpreting genome-wide expression profiles. Proc Natl Acad Sci U S A. 2005;102:15545-50.

4. Chen YP, Wang YQ, LV JW, et al. Identification and validation of novel microenvironment-based immune molecular subgroups of head and neck squamous cell carcinoma: implications for immunotherapy. Ann Oncol 2019:30:68-75.

5. Thorsson V, Gibbs DL, Brown SD, et al. The immune landscape of cancer. Immun ity 2018:48:812-30.

6. Ayers $M$, Lunceford J, Nebozhyn $M$, et al. IFN-gamma-related mRNA profile predicts clinical response to PD-1 blockade. J Clin Invest 2017;127:2930-40.

Ethics Approval The use of clinical specimens in this study has been approved by the Medical Ethics Committee, Faculty of Dentistry, University of Malaya [DF OS1624/0073(L)], and The National Medical Research Register, Malaysia [NMRR-161764-32566 (IIR)].

http://dx.doi.org/10.1136/jitc-2021-SITC2021.911 